\title{
EDITORIAL
}

\section{Immune system and cardiovascular disease}

\begin{abstract}
The aim of this
Collection is to highlight the role of inflammatory

processes in cardiovascular disease
\end{abstract}

Innate and adaptive immune responses have an essential role in the development and progression of many cardiovascular diseases. The concept of atherosclerosis - the primary cause of coronary artery disease, stroke, and peripheral vascular disease - as a chronic inflammatory disease is widely accepted. Inflammation is also involved in the pathophysiology of atrial fibrillation, the most common cardiac arrhythmia. Defects in the resolution of inflammation promote the progression to vulnerable plaque in atherosclerosis, and altered immune responses can lead to cardiac remodelling after myocardial infarction. To highlight the importance of the immune system in the pathophysiology of cardiovascular diseases, Nature Reviews Cardiology brings together a Collection ${ }^{1}$ of Reviews and news articles that summarize our current knowledge and recent advances in this field.

Three Reviews in this Collection focus on specific immune cells and their role in cardiovascular diseases.

Chinetti-Gbaguidi and colleagues review the diverse range of macrophage phenotypes in atherosclerotic lesions and their roles in plaque progression and stability. Shi et al. focus on mast cells, which are involved in the pathogenesis of many cardiometabolic diseases, including atherosclerosis, aortic aneurysm, valvular disease, obesity, and diabetes mellitus. Accumulating evidence indicates that regulatory $\mathrm{T}$ cells $\left(\mathrm{T}_{\text {reg }}\right)$ - which regulate T-cell responses and induce antiinflammatory macrophages - might have an important role in protecting against cardiovascular disease. The latest discoveries on the role of $\mathrm{T}_{\text {reg }}$ cells in several cardiovascular disorders, such as atherosclerosis, hypertension, and myocardial infarction, are discussed by Meng and colleagues in their Review.

Great progress in understanding the role of inflammatory processes in the pathogenesis of a diverse range of cardiovascular diseases has been made in recent years. As discussed by $\mathrm{Hu}$ and colleagues, accumulating evidence suggests that inflammation is involved in the pathophysiology of atrial fibrillation and, in turn, atrial fibrillation can induce inflammation. MicroRNAs, as highlighted by Schober et al. in their Review, have been implicated in the mechanisms underlying chronic inflammatory processes in atherosclerosis. Myocarditis, a frequent cause of dilated cardiomyopathy and sudden cardiac death, typically results from a maladaptive hyperimmune response triggered by viral infection, and in most patients with recurrent pericarditis an immune-mediated pathogenesis is often presumed. The diagnosis and therapeutic options for these conditions are reviewed by Pollack et al. and Imazio et al., respectively.

Technical advances in imaging atherosclerotic plaques have provided unique opportunities in both research and clinical practice. Novel modalities for molecular imaging of human coronary arteries in vivo, such as the dual-modality optical coherence tomography and near-infrared autofluorescence, discussed in a News \& Views article by Psaltis and Nicholls, have greatly enhanced imaging resolution and the capacity to detect and quantify pathological processes within the plaque. New PET tracers have emerged for tracking plaque inflammation, hypoxia, or calcification (summarized by Tarkin and colleagues in their Review), which can potentially be used as a markers of plaque vulnerability for risk stratification of patients.

Another issue that has received much attention over the past few years is therapies that target the immune response for the prevention and treatment of cardiovascular diseases. As described by Bäck and Hansson in their Review, therapies that are currently used or are under evaluation for other diseases are being explored as potential anti-inflammatory therapies for atherosclerosis. In his Review, Frangogiannis highlights potential immune-targeted therapies to counterbalance the destructive effects of cardiac remodelling after myocardial damage. Anti-inflammatory therapeutic strategies also have the potential to be used in atrial fibrillation, viral myocarditis, and recurrent pericarditis.

Improving our understanding of the inflammatory processes involved in the pathophysiology of cardiovascular diseases can lead to new opportunities for the management of these patients. Current therapeutic approaches to atherosclerosis and myocardial infarction focus on risk-factor reduction and revascularization; the aim of this Collection is to highlight the role of inflammatory processes in cardiovascular disease and to identify novel anti-inflammatory therapeutic approaches.

1. Nature Reviews Cardiology. Immune system and cardiovascular disease (Collection), http://www. nature.com/immunecvd (2016). 